\title{
APPROXIMATE FIXED POINTS ON ALMOST CONVEX SETS
}

\author{
J. E. C. LOPE, R. M. REY, M. ROQUE AND P. W. SY
}

\begin{abstract}
In this paper, we deduce a maximal element theorem on multimaps and an approximate fixed point theorem on almost convex sets. This generalizes the well-known Himmelberg fixed point theorem and also unifies recent results of Park and Tan [14] and Sy and Park [16].
\end{abstract}

The celebrated Knaster-Kuratowski-Mazurkiewicz (simply KKM) principle is a versatile tool to obtain fixed point theorems on convex subsets of topological vector spaces. For examples, Park and Tan ([13], [14]) gave simple proofs of the generalizations of fixed point theorems due to Schauder, Tychonoff, and Himmelberg by applying the KKM principle directly.

In an earlier work of Sy and Park [16], the KKM principle is applied to obtain a new non-compact version of the Fan-Browder fixed point theorem, from which an approximate fixed point theorem is deduced. In this paper, we follow the method of [16] and obtain a maximal element theorem and an approximate fixed point theorem which unify those in Park and Tan ([13], [14]) and Sy and Park [16].

A multimap (or simply, a map) $F: X \multimap Y$ is a function from a set $X$ into the power set $2^{Y}$ of a set $Y$; that is, a function with the values $F(x) \subset Y$ for $x \in X$ and the fibers $F^{-}(y):=\{x \in X: y \in F(x)\}$ for $y \in Y$. For $A \subset X$, let $F(A):=\bigcup\{F(x): x \in A\}$.

For a set $D$, let $\langle D\rangle$ denote the set of nonempty finite subsets of $D$.

Let $X$ be a subset of a vector space and $D$ a nonempty subset of $X$. We call $(X, D)$ a convex space if $\operatorname{co} D \subset X$ and $X$ has a topology that induces the Euclidean topology on the convex hulls of any $N \in\langle D\rangle$; see [5], [6]. If $X=D$ is convex, then $X=(X, X)$ becomes a convex space in the sense of Lassonde [4]. If $X$ is compact, then the convex space $(X, D)$ is said to be compact. Every nonempty convex subset $X$ of a topological vector space is a convex space with respect to any nonempty subset $D$ of $X$, and the converse is known to be not true.

The following version of the Knaster-Kuratowski-Mazurkiewicz (simply, KKM) theorem for convex spaces is known.

Theorem 1. Let $(X, D)$ be a convex space and $F: D \multimap X$ a multimap such that

(1) $F(z)$ is open $[$ resp. closed $]$ for each $z \in D$; and

Received March 14, 2003.

2000 Mathematics Subject Classification. 47H04, 47H10, 52H07, 54C60, 54H25.

Key words and phrases. KKM maps, almost convex spaces, the Himmelberg fixed point theorem. 
(2) $F$ is a KKM map (that is, coN $\subset F(N)$ for each $N \in\langle D\rangle$ ).

Then $\{F(z)\}_{z \in D}$ has the finite intersection property. (More precisely, for any $N \in\langle D\rangle$, we have $\operatorname{co} N \cap\left[\bigcap_{z \in N} F(z)\right] \neq \emptyset$.)

The closed version is essentially due to Fan [1] and the open version is motivated from the works of Kim [3] and Shih-Tan [15], who showed that the original KKM theorem holds for open valued KKM maps on a simplex. Later, Lassonde [5] showed that the closed and open versions of Theorem 1 can be derived from each other. More general versions of Theorem 1 were recently known; for example, see Park ([10] - [12]).

From Theorem 1, Sy and Park [16] obtained the following.

Theorem 2. Let $(X, D)$ be a convex space and $P: X \multimap D$ a multimap. If there exist $z_{1}, z_{2}, \ldots, z_{n} \in D$ and nonempty open [resp. closed] subsets $G_{i} \subset P^{-}\left(z_{i}\right)$ for each $i=1,2, \ldots, n$ such that $\operatorname{co}\left\{z_{1}, z_{2}, \ldots, z_{n}\right\} \subset \bigcup_{i=1}^{n} G_{i}$, then the map coP $: X \multimap X$ has a fixed point $x_{0} \in X$ (that is, $\left.x_{0} \in \operatorname{coP}\left(x_{0}\right)\right)$.

From Theorem 2, we have the following.

Theorem 3. Let $(X, D)$ be a compact convex space and $P: X \multimap D$ a map such that

(1) $x \notin \operatorname{co} P(x)$ for all $x \in X$; and

(2) $P^{-}(y)$ is open for all $y \in D$.

Then there exists an $\bar{x} \in X$ such that $P(\bar{x})=\emptyset$.

Proof. Suppose $P(x) \neq \emptyset$ for all $x \in X$. Then $X=\bigcup_{y \in D} P^{-}(y)$. Since $X$ is compact, $X=\bigcup_{y \in N} P^{-}(y)$ for some $N \in\langle D\rangle$. Then by Theorem 2, co $P$ has a fixed point, which contradicts $(1)$.

For $X=D$, Theorem 3 reduces to theorems of Toussaint [17] and Yannelis and Prabhakar [18] on the existence of maximal elements.

A nonempty subset $Y$ of a topological vector space $E$ is said to be almost convex if for any neighborhood $V$ of the origin $O$ in $E$ and for any finite set $\left\{y_{1}, y_{2}, \ldots, y_{n}\right\} \subset Y$, there exists a finite set $\left\{z_{1}, z_{2}, \ldots, z_{n}\right\} \subset Y$ such that, for each $i \in\{1,2, \ldots, n\}$, we have $z_{i}-y_{i} \in V$ and $\operatorname{co}\left\{z_{1}, z_{2}, \ldots, z_{n}\right\} \subset Y$; see [2].

From Theorem 2, we deduce the following approximate fixed point theorem.

Theorem 4. Let $X$ be a subset of a topological vector space $E$ and $Y$ an almost convex dense subset of $X$. Let $F: X \multimap X$ be a lower [resp. upper] semi-continuous map such that (1) $F$ has nonempty values, (2) $F(y)$ is convex for all $y \in Y$, and (3) $F(X)$ is totally bounded. Then for every open [resp. closed] convex neighborhood $V$ of the origin $O$ of $E$, there exists a point $x_{V} \in Y$ such that

$$
F\left(x_{V}\right) \cap\left(x_{V}+V\right) \neq \emptyset .
$$

Proof. Let $V$ be the given symmetric neighborhood of $O$ in $E$. Then there exists a neighborhood $U$ of $O$ such that $U+U \subset V$. Since $F(X)$ is totally bounded in $X$, there 
exists a finite subset $\left\{x_{1}, x_{2}, \ldots, x_{n}\right\} \subset F(X)$ such that $F(X) \subset \bigcup_{i=1}^{n}\left(x_{i}+U\right)$. Moreover, since $Y$ is almost convex and dense in $X$, there exists a finite subset $D:=\left\{y_{1}, y_{2}, \ldots, y_{n}\right\}$ of $Y$ such that $x_{i}-y_{i} \in U$ for each $i \in\{1,2, \ldots, n\}$ and $Z:=\operatorname{co}\left\{y_{1}, y_{2}, \ldots, y_{n}\right\} \subset Y$.

Since $x_{i}+U=y_{i}+\left(x_{i}-y_{i}\right)+U \subset y_{i}+U+U \subset y_{i}+V$, we have $F(Z) \subset F(X) \subset$ $\bigcup_{i=1}^{n}\left(y_{i}+V\right)$. Define a map $P: Z \multimap D$ by $P(z):=(F(z)-V) \cap D$ for $z \in Z$. Then each $P(z)$ is nonempty. Note that for each $y \in D$, we have

$$
\begin{aligned}
P^{-}(y) & =\{z \in Z: y \in P(z)\} \\
& =\{z \in Z: y \in(F(z)-V) \cap D\} \\
& =\{z \in Z: F(z) \cap(y+V) \neq \emptyset\} .
\end{aligned}
$$

If $F$ is lower semi-continuous and $V$ is open, then each $P^{-}(y)$ is open in $Z$. If $F$ is upper semi-continuous and $V$ is closed, then each $P^{-}(y)$ is closed in $Z$.

Note that for each $z \in Z$, we have a $y \in D$ such that $z \in P^{-}(y)$. Therefore, $Z \subset Y=$ $\bigcup_{y \in D} P^{-}(y)$. Hence, by Theorem 2, co $P: Z \multimap Z$ has a fixed point $x_{V} \in Z \subset Y$, that is, $x_{V} \in \operatorname{co} P\left(x_{V}\right)$. Since $x_{V} \in Z \subset Y, F\left(x_{V}\right)$ is convex and hence, $x_{V} \in \operatorname{co} P\left(x_{V}\right) \subset$ $\operatorname{co}\left[\left(F\left(x_{V}\right)-V\right) \cap D\right] \subset\left(F\left(x_{V}\right)-V\right) \cap Z$, which readily implies $F\left(x_{V}\right) \cap\left(x_{V}+V\right) \neq \emptyset$.

If $X=Y$ is almost convex, then Theorem 4 improves Theorem 5 obtained in Sy and Park [16].

We now deduce the following result due to Park and Tan [14].

Theorem 5. (Park and Tan [14], Theorem 1) Let X be a subset of a locally convex Hausdorff topological vector space $E$ and $Y$ an almost convex dense subset of $X$. Let $T: X \multimap X$ be a compact upper semi-continuous multimap with nonempty closed values such that $T(y)$ is convex for all $y \in Y$. Then $T$ has a fixed point $x_{0} \in X$; that is, $x_{0} \in T\left(x_{0}\right)$.

Proof. For each neighborhood $V$ of $O$, there exist $x_{V}, y_{V} \in X$ such that $y_{V} \in T\left(x_{V}\right)$ and $y_{V} \in x_{V}+V$. Since $T(X)$ is relatively compact, we may assume that the net $\left\{y_{V}\right\}$ converges to some $x_{0} \in X$. Since $E$ is Hausdorff, the net $\left\{x_{V}\right\}$ also converges to $x_{0}$. Because $T$ is upper semi-continuous with closed values, the graph of $T$ is closed in $X \times T(X)$ and hence we have $x_{0} \in T\left(x_{0}\right)$. This proves the theorem.

In particular, for $X=Y$, we obtain

Theorem 6. (Park and Tan [14], Theorem 2) Let X be an almost convex subset of a locally convex Hausdorff topological vector space. Then any compact upper semicontinuous multimap $T: X \multimap X$ with nonempty closed convex values has a fixed point in $X$.

If $X$ itself is convex, Theorem 6 reduces to the Himmelberg fixed point theorem.

From Theorem 4 or from Theorem 5 , we obtain 
Theorem 7. (Park and Tan [13], Theorem 1) Let $X$ be an almost convex subset of a locally convex Hausdorff topological vector space $E$ and $f: X \multimap X$ a compact continuous map. Then $f$ has a fixed point.

Further, from the lower semi-continuous case of Theorem 4, we deduce the following.

Theorem 8. Let $X$ be a subset of a topological vector space and $Y$ an almost convex dense subset of $X$. Let $F: X \multimap X$ be a multimap such that

(1) $F(x)$ is nonempty for each $x \in X$;

(2) $F(y)$ is convex for each $y \in Y$;

(3) $F^{-}(z)$ is open for each $z \in X$; and

(4) $F(X)$ is totally bounded.

Then for any convex neighborhood $V$ of $O$ in $E$, there exists a point $x_{V} \in X$ such that $F\left(x_{V}\right) \cap\left(x_{V}+V\right) \neq \emptyset$.

Proof. Simply $F$ is lower semi-continuous.

If $X=Y$ is convex, then Theorem 8 reduces to Sy and Park ([16], Theorem 7).

\section{References}

[1] Ky Fan, A generalization of Tychonoff's fixed point theorem, Math. Ann. 142(1961), 305-310.

[2] C. J. Himmelberg, Fixed points of compact multifunctions, J. Math. Anal. Appl. 38(1972), 205-207.

[3] W. K. Kim, Some applications of the Kakutani fixed point theorem, J. Math. Anal. Appl. 121(1987), 119-122.

[4] M. Lassonde, On the use of KKM multifunctions in fixed point theory and related topics, J. Math. Anal. Appl. 97(1983), 151-201.

[5] M. Lassonde, Sur le principe KKM, C. R. Acad. Sci. Paris, Série I, 310(1990), 573-576.

[6] Sehie Park, Foundations of the KKM theory via coincidences of composites of upper semicontinuous maps, J. Korean Math. Soc. 31(1994), 493-519.

[7] Sehie Park, Remarks on a fixed point problem of Ben-El-Mechaiekh, in: Nonlinear Analysis and Convex Analysis (Proc. NACA'98, Niigata, Japan, Jul. 28-31, 1998), pp. 79-86, World Sicentific, Singapore, 1999.

[8] Sehie Park, Ninety years of the Brouwer fixed point theorem, Vietnam J. Math. 27 (1999), 187-222.

[9] Sehie Park, The Knaster-Kuratowski-Mazurkiewicz theorem and almost fixed points, Topological Methods in Nonlinear Anal., J. of the Julius Schauder Center, 16(2000), 195-200.

[10] Sehie Park, Elements of the KKM theory for generalized convex spaces, Korean J. Comp. Appl. Math. 7(2000), 1-28.

[11] Sehie Park, Remarks on topologies of generalized convex spaces, Nonlinear Funct. Anal. Appl. 5(2000), 67-79.

[12] Sehie Park, Fixed point theorems in locally G-convex spaces, Nonlinear Anal. 48(2002), 869-879. 
[13] Sehie Park and Do Hong Tan, Remark on the Schauder-Tychonoff fixed point theorem, Vietnam J. Math. 28(2000), 51-64.

[14] Sehie Park and Do Hong Tan, Remark on Himmelberg-Idzik's fixed point theorem, Acta Math. Viet. 25(2000), 285-289.

[15] M.-H. Shih and K.-K. Tan, Covering theorems of convex sets related to fixed point theorems, in : Nonlinear and Convex Analysis (Proc. in Honor of Ky Fan), pp. 235-244, Marcel Dekker, Inc., New York-Basel, 1987.

[16] Polly W. Sy and Sehie Park, The KKM maps and fixed point theorems in convex spaces, Tamkang J. 34, 2003.

[17] S. Toussaint, On the existence of equilibria in economies with infinitely many commodities and without ordered preferences, J. Econ. Th. 33 (1984), 98-115.

[18] N. C. Yannelis and N. D. Prabhakar, Existence of maximal elements and equilibria in linear topological spaces, J. Math. Econ. 12 (1983), 233-235.

Department of Mathematics, College of Science, University of the Philippines, Diliman, Quezon City, Philippines.

E-mail: pweesy@i-manila.com.ph 